\title{
Emergency department confirmation of peripherally inserted central catheter placement by bedside ultrasound
}

\author{
Cindy W. Chan $•$ Michael J. Lambert
}

Received: 29 July 2009 / Accepted: 1 September 2009 / Published online: 26 September 2009

(C) Springer-Verlag London Ltd 2009

A 28-year-old woman presented to the emergency department (ED) for acute respiratory distress from an extended care facility, where she was rehabilitating from a traumatic brain injury (TBI) and remained comatose. On arrival, the patient was tachycardic, with a sinus rhythm on monitor at $140 \mathrm{bpm}$. She was placed on a ventilator with settings as previously recorded at the nursing home. Blood pressure was $84 / 56$ and temperature $37.8^{\circ} \mathrm{C}$. Physical exam revealed good breath sounds bilaterally, heart was tachycardic without murmur, abdomen was scaphoid without guarding, and skin was warm to touch. Her only venous access was a peripherally inserted central catheter (PICC) line in her right antecubital fossa.

A bedside ultrasound was performed by an emergency physician to confirm placement of the patient's PICC line. Verification of placement can rapidly be determined with ultrasound by following the peripheral vein of insertioncommonly cephalic, basilic, or brachial-through to the subclavian vein. A quick look at the neck and heart will also ensure the catheter tip has not inadvertently migrated into the internal jugular (IJ) vein or right side of the heart.

Transverse views of the patient's right basilic vein and axillary vein (Fig. 1), continued with a sagittal view of the subclavian vein (Fig. 2), demonstrated a PICC line in place. A transverse view of the right IJ vein was also taken to confirm that the PICC had not inappropriately advanced to that location. Once line placement was confirmed with bedside ultrasound, the PICC was able to be used without the delay of chest X-ray confirmation.

\footnotetext{
C. W. Chan $(\bowtie) \cdot$ M. J. Lambert

Department of Emergency Medicine,

Advocate Christ Medical Center,

4440 West 95th Street,

Oak Lawn, IL 60453, USA

e-mail: cindychanmd@gmail.com

M. J. Lambert

e-mail: mlambert62@mac.com
}

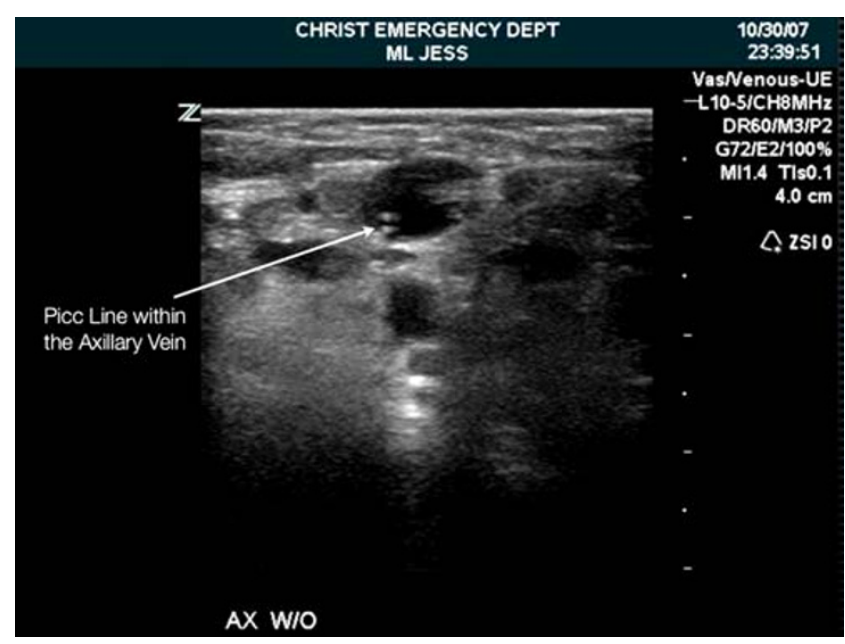

Fig. 1 Transverse view of axillary vein

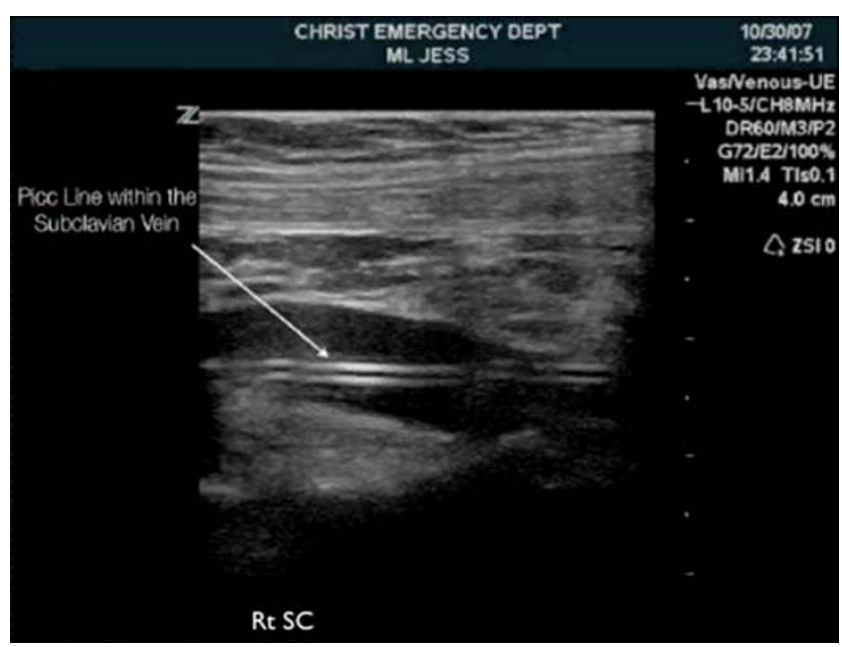

Fig. 2 Sagittal view of subclavian vein 